\title{
NON-DECREASING STEP RESPONSES WHOSE TRANSFER FUNCTIONS ARE SUBCLASS $K *$
}

BY

\author{
ARMEN H. ZEMANIAN
}

New York University

Introduction. This paper is concerned with the intersection of two classes of rational system functions. The first class was called the subclass $k$ in previous papers and its functions constitute generalizations of the positive-real functions [1-4]. Functions of this type are quite common in electric network theory. For instance, the reciprocals of Hurwitz polynomials are members of this class and they are used quite frequently in network synthesis [5-7]. The transfer functions of the series-shunt peaking network and the Dietzold network [8] are also subclass $k$ functions. Or again, a minimum-phase function having a monotonic decreasing** phase function is a subclass $k$ function [1]. The unit step responses of such functions need not be monotonic.

The second class is comprised of those rational system functions whose corresponding unit step responses are non-decreasing [9-12]. Such functions are of interest in those applications where an overshoot in the step response is to be avoided. For instance, this is often the case in measuring apparatus.

It has been shown previously that certain restrictions exist on the step response of any subclass $k$ function. If, in addition, this response is known to be monotonic increasing, it is possible to strengthen these restrictions. This is one of the objectives of this paper. Of course, the results that will be developed here are useful only if it is known that the step response is monotonic increasing. Various sets of sufficient conditions that insure this are given in [9-12].

The second objective is the following. In [1], lower bounds on the rise time and the settling time of the unit step response corresponding to a subclass $k$ transfer function were obtained. These lower bounds were the envelopes of families of straight lines wherein the coefficients of the straight line expressions contained certain infinite series. Through numerical computation these series were summed and then the envelopes were determined by graphical means. Results were obtained for values of $k$ that ranged from one to five. In this paper the infinite series are summed into closed forms and parametric equations for the envelopes, which hold for all positive integer values of $k$, are developed. Such expressions for non-decreasing step responses are given within the body of the paper. The parametric equations for the envelopes when the step responses need not be monotonic (i.e., such as those of [1]) are given in the appendix.

Finally, having such results available, one may formulate lower bounds (in terms of definite integrals of certain functions) on the moments of non-decreasing step responses having subclass $k$ transfer functions. This additional result is also of significance in probability theory wherein moments have been used to describe the probability distribution function. In this case a distribution function corresponds to the non-decreasing

* Received February 8, 1960.

**Here, the phase angle is considered positive when a steady-state response leads a sinusoidal input. 


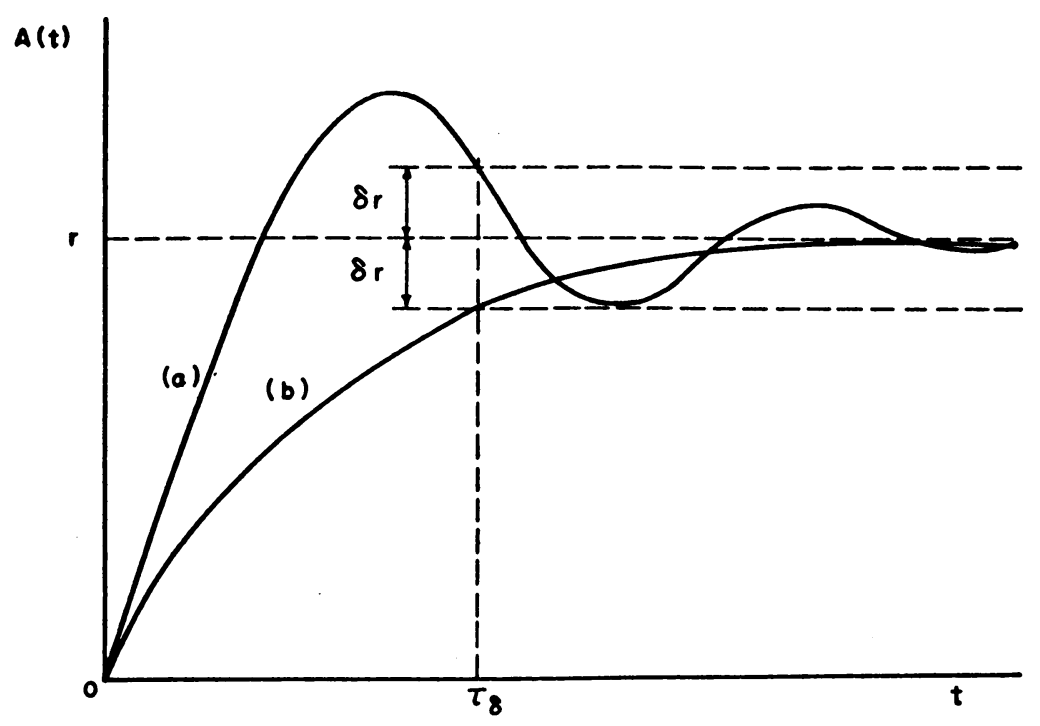

Fig. 1. Illustration of the settling time $\tau_{\delta}$ (a) for a unit step response that has overshoot and (b) for a unit step response that is non-decreasing.

step response and its characteristic function corresponds to the system function. The rationality restriction on the characteristic function or on the system function may be dropped if the subclass $k$ functions are defined as in [3].

The notations used here will be the same as those of [1] and this paper should be read as a sequel to it.

Definitions. Let $Z(s)$ be the following rational system function of the complex variable $s=\sigma+j \omega$, where all the coefficients are real numbers

$$
Z(s)=K \frac{s^{n}+a_{n-1} s^{n-1}+\cdots+a_{0}}{s^{m}+b_{m-1} s^{m-1}+\cdots+b_{0}}=K \frac{N(s)}{D(s)} .
$$

Furthermore, let $K>0, k=m-n \geq 1, Z(0)=K a_{0} / b_{v}=r$, and let $D(s)$ be a Hurwitz polynomial. The real and imaginary parts of $Z(j \omega)$ will be denoted by $R(\omega)$ and $I(\omega)$, respectively. Under these conditions, the response $A(t)$ of the initially quiescent system to a unit step input applied at time $t=0$ will be a continuous function for all $t . A(t)$ is related to $Z(s)$ through the following Laplace-Stieltjes transform

$$
Z(s)=\int_{0}^{\infty} e^{-s t} d A(t) .
$$

The subclass $k$ is defined as follows. $Z(s)$ will be called a subclass $k$ function if the following conditions are satisfied. For $k$ odd, $R(\omega)$ has $k-1$ changes of sign in $-\infty<$ $\omega<\infty$ and $R(\omega)$ is positive in the neighborhood of $\omega=0$. For $k$ even, $I(\omega)$ has $k-1$ changes of sign in $-\infty<\omega<\infty$ and $d I / d \omega$ is negative in the neighborhood of $\omega=0$.

In the following analysis we shall assume that the final value $r$ of the unit step response is a positive quantity. Another quantity that will be considered is the settling time to $\delta$ which is denoted by $\tau_{\delta}$. This settling time is the least time beyond which $A(t)$ remains within the bounds $(1 \pm \delta) r$ where $0 \leq \delta \leq 1$. A particular settling time is illustrated in Fig. 1 for a unit step response that has overshoot and for another unit 


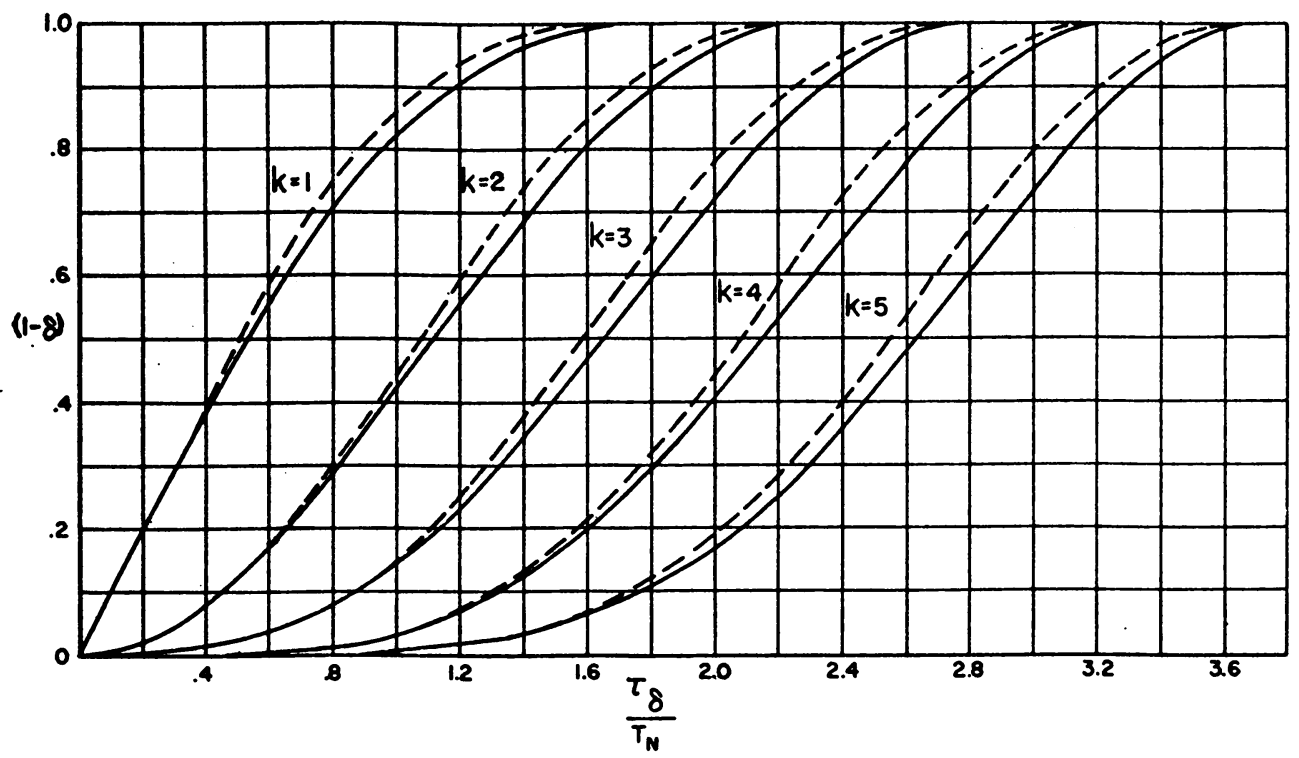

Fra. 2. The lower bounds on the settling times for the unit step responses of the subclass $k$ functions. The solid curves are the lower bounds in the case where the unit step responses are non-decreasing. The dotted curves are for the general case where the unit step responses need not be monotonic.

step response that is non-decreasing. When $A(t)$ is non-decreasing, any value of time $t$ is a settling time $\tau_{\delta}$ where $\delta=1-A(t) / r$.

Upper bounds on non-decreasing step responses. If a system function is a subclass $k$ function, the settling time to $\delta$ cannot be less than certain lower bounds given by the dotted curves of Fig. 2 [1]. That is, given the unit step response of a subclass $k$ function, any deviation $\delta r(0 \leq \delta \leq 1)$ from the final value $r$ will determine a settling time $\tau_{\delta}$; the point, whose coordinates are $\tau_{\delta} / T_{N}$ and $(1-\delta)$, must lie to the right of the dotted curve for the given $k$. In Fig. 2 the time scale is normalized by the factor $T_{N}$ which is given by

$$
T_{N}=\left[\frac{r}{K}\right]^{1 / k}
$$

For non-decreasing step responses this result can be strengthened. The new lower bounds on the settling time are given by the solid curves of Fig. 2. They may be derived in a fashion similar to the development of the dotted curves. In fact, the first parts of the two derivations are precisely the same and will not be repeated in this paper. This section gives the derivation of the solid curves starting at the point where the development diverges from that of the dotted curves.

This result and the monotonicity of $A(t)$ together imply a still stronger restriction. Any time $t$ is a settling time $\tau_{\delta}$ for non-decreasing step responses. Hence, for a given $k$ the corresponding solid curve of Fig. 2 is an upper bound on a non-decreasing $A(t)$. The upper bounds that were previously shown to exist [1] on the entire unit step responses of all subclass $k$ functions are given by the dotted curves of Fig. 3. For comparison, the new upper bounds are repeated in this figure as the solid curves and a substantial improvement may be noted. The examples of the next section indicate that these upper bounds 


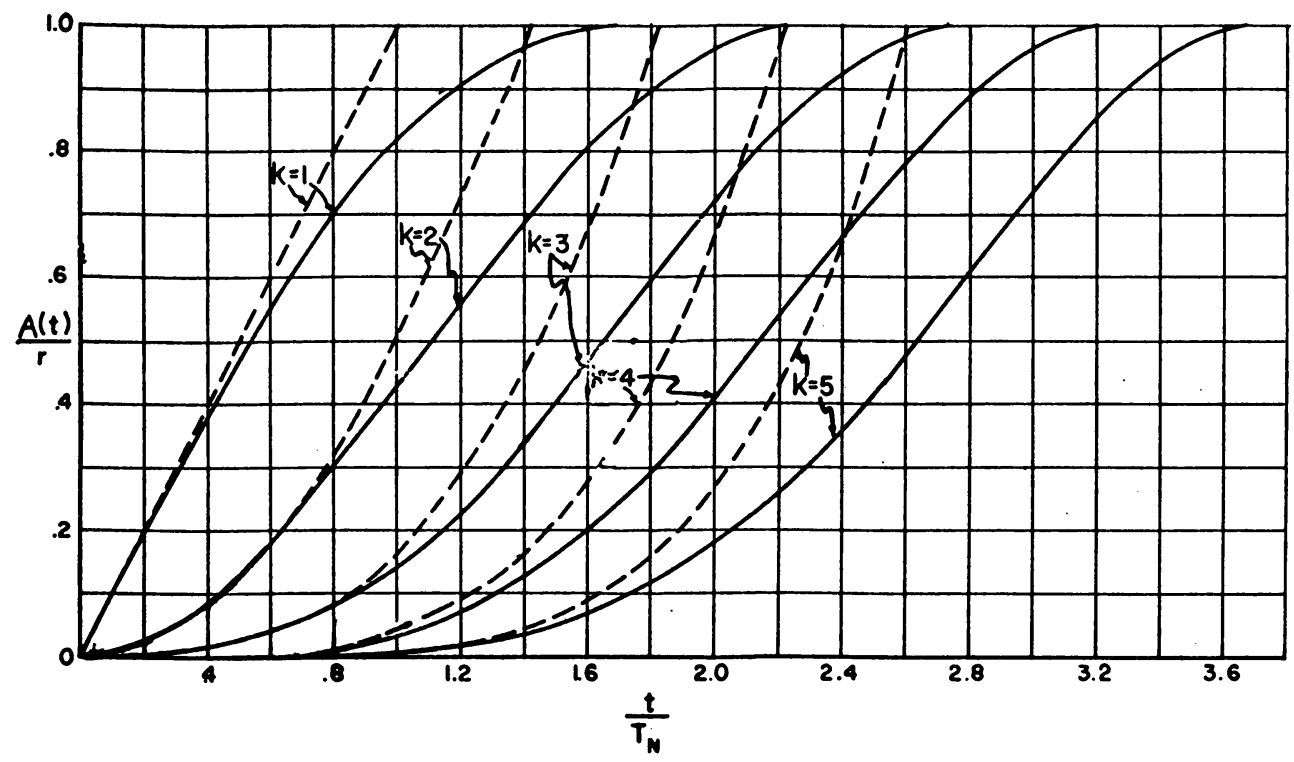

Fra. 3. The upper bounds on the unit step responses of the subclass $k$ functions. The solid curves are the upper bounds in the case where the unit step responses are non-decreasing. The dotted curves are for the general case where the unit step responses need not be monotonic.

are fairly close to being best possible when $k=1$ except possibly in the neighborhood of the final value line.

This conclusion may be stated as follows.

Theorem. Let $Z(s)$ be a subclass $k$ function, let the corresponding $A(t)$ be non-decreasing, let $B$ be the upper bound on $A(t) / r$, and let

$$
\xi=\frac{1}{k !}\left[\frac{t}{T_{N}}\right]^{k} \text {. }
$$

Then the parametric equations for the upper bound on $A(t)$ are given by (4), (5), and (6) for $k$ odd and by (4), (7), and (8) for $k$ even, where $0<y<1$.

$$
\begin{gathered}
\xi=2 \sum_{\mu=0}^{(k-3) / 2} y^{k-2 \mu}\left[1-\frac{1}{2^{k-2 \mu-1}}\right]\left[1-\frac{k-2 \mu}{1-\pi y \operatorname{ctn} \pi y}\right] L i_{k-2 \mu}(1) \\
\quad+\frac{y^{2}}{2(1-\pi y \operatorname{ctn} \pi y)}\left\{\pi \operatorname{ctn} \pi y[\Omega(y)-4 \log 2]+\Omega^{\prime}(y)\right\}, \\
B=\frac{\sin \pi y}{\pi(1-\pi y \operatorname{ctn} \pi y)}\left\{-2 \sum_{\mu=0}^{(k-3) / 2}(k-2 \mu) y^{k-2 \mu-1}\left[1-\frac{1}{2^{k-2 \mu-1}}\right] L i_{k-2 \mu}(1)\right. \\
\left.-2 \log 2+\frac{\Omega(y)}{2}+\frac{y \Omega^{\prime}(y)}{2}\right\}, \\
\xi=1+2 \sum_{\mu=0}^{(k-2) / 2} y^{k-2 \mu}\left[1-\frac{1}{2^{k-2 \mu-1}}\right]\left[1-\frac{k-2 \mu}{1-\pi y \operatorname{ctn} \pi y}\right] L i_{k-2 \mu}(1), \\
B=1-\frac{2 \sin \pi y}{\pi(1-\pi y \operatorname{ctn} \pi y)} \sum_{\mu=0}^{(k-2) / 2}(k-2 \mu) y^{k-2 \mu-1}\left[1-\frac{1}{2^{k-2 \mu-1}}\right] L i_{k-2 \mu}(1),
\end{gathered}
$$


where

$$
\begin{gathered}
\Omega(y)=\Psi\left(1-\frac{y}{2}\right)-\Psi\left(\frac{1}{2}-\frac{y}{2}\right)+\Psi\left(1+\frac{y}{2}\right)-\Psi\left(\frac{1}{2}+\frac{y}{2}\right) \\
\Omega^{\prime}(y)=\frac{1}{2}\left[-\Psi^{\prime}\left(1-\frac{y}{2}\right)+\Psi^{\prime}\left(\frac{1}{2}-\frac{y}{2}\right)+\Psi^{\prime}\left(1+\frac{y}{2}\right)-\Psi^{\prime}\left(\frac{1}{2}+\frac{y}{2}\right)\right] .
\end{gathered}
$$

In (5) and (6) the summations on $\mu$ do not exist if $k=1$. Furthermore, $L i_{p}(1)$ is the polylogarithm of order $p$ and argument one and $\Psi(x)$ and $\Psi^{\prime}(x)$ are the digamma function and its derivative, respectively.

Note. Tables of $L i_{\nu}(x)$ and of $\Psi(x)$ and $\Psi^{\prime}(x)$ may be found in [13] and [14], respectively. It should also be noted that this theorem holds for a wider class of transfer functions (i.e., for those functions that satisfy inequalities (8) and (9) in [1]).

Proof. As mentioned previously, the first part of the proof for $k \geq 2$ is exactly as given in [1] up to expression (35) and this part will not be repeated here. For $k=1$, see [15]. Expression (35) of [1] is the following inequality wherein $0 \leq y<1$. (Note that the upper limit $N$ in the summation of expression (35) of [1] should be replaced by $\infty$.)

$$
A(t) \leq \frac{\sin \pi y}{\pi y}\left[\frac{K t^{k}}{k !}+2 y^{k+2} \sum_{p=1}^{\infty} \frac{(-1)^{p+1} A(p t / y)}{p^{k}\left(p^{2}-y^{2}\right)}\right] .
$$

Now those values for $A(p t / y)$ that maximize the right-hand side of (11) nust be found. Since $0 \leq y<1,(\sin \pi y) / \pi y$ is positive and the coefficients of the $A(p t / y)$ alternate in sign and decrease in magnitude, the first coefficient being positive. Furthermore, the $A(p t / y)$ are non-decreasing and no larger than $r$. Hence, for any given, permissible set of values for $A(\nu t / y),(\nu=1,3,5, \cdots)$, the summation will assume its maximum value when $A[(\nu+1) t / y]$ is set equal to $A(\nu t / y)$. Under this condition the summation in (11) may be rewritten as

$$
2 y^{k+2} \sum_{\substack{\nu=1 \\ \nu \text { odd }}}^{\infty} A\left(\frac{\nu t}{y}\right)\left\{\frac{1}{\nu^{k}\left(\nu^{2}-y^{2}\right)}-\frac{1}{(\nu+1)^{k}\left[(\nu+1)^{2}-y^{2}\right]}\right\} .
$$

The coefficients of $A(\nu t / y)$ in (12) are all positive so that this expression and hence the right-hand side of (11) are maximized by setting $A(\nu t / y)=r$.

Inserting the quantities $B$ and $\xi$, which are defined in the hypothesis, and rearranging the result, (12) becomes the following equality.

$$
\xi=\frac{\pi y}{\sin \pi y} B-2 y^{k+2} \sum_{p=1}^{\infty} \frac{(-1)^{p+1}}{p^{k}\left(p^{2}-y^{2}\right)} .
$$

Let

$$
C(y)=\frac{\pi y}{\sin \pi y}
$$

and let

so that (13) becomes

$$
E(y)=2 y^{k+2} \sum_{p=1}^{\infty} \frac{(-1)^{p+1}}{p^{k}\left(p^{2}-y^{2}\right)}
$$

$$
\xi=C(y) B-E(y) .
$$


Now $E(y)$ may be summed by first expanding each term of the series into partial fractions.

$$
\frac{1}{p^{k}\left(p^{2}-y^{2}\right)}=\frac{1}{2 y} \sum_{\eta=0}^{k-1}\left[1+(-1)^{\eta}\right]\left[\frac{-1}{y^{\eta+1} p^{k-\eta}}\right]+\frac{1}{2 y^{k+1}}\left[\frac{1}{p-y}-\frac{(-1)^{k}}{p+y}\right] .
$$

Summing the last two partial fractions in (17) according to (15), we have from [16; $p$. 20, formula 1.7.4.(33)]

$$
\begin{gathered}
\sum_{p=1}^{\infty}(-1)^{p+1}\left[\frac{1}{p-y}+\frac{1}{p+y}\right]=\frac{\Omega(y)}{2} \\
\sum_{p=1}^{\infty}(-1)^{p+1}\left[\frac{1}{p-y}-\frac{1}{p+y}\right] \\
=\frac{1}{2}\left[\Psi\left(1-\frac{y}{2}\right)-\Psi\left(\frac{1}{2}-\frac{y}{2}\right)-\Psi\left(1+\frac{y}{2}\right)+\Psi\left(\frac{1}{2}+\frac{y}{2}\right)\right] .
\end{gathered}
$$

The right-hand side of the last equation may be simplified by using $[16 ; p .16$, formulas 1.7.1.(11)]

$$
\sum_{p=1}^{\infty}(-1)^{p+1}\left[\frac{1}{p-y}-\frac{1}{p+y}\right]=\frac{\pi}{2}\left[\tan \frac{\pi y}{2}+\operatorname{ctn} \frac{\pi y}{2}\right]-\frac{1}{y}=\frac{\pi}{\sin \pi y}-\frac{1}{y} .
$$

Moreover,

$$
\sum_{p=1}^{\infty} \frac{(-1)^{p+1}}{p}=\log 2
$$

and, for $k=2,3,4, \cdots$,

$$
\sum_{p=1}^{\infty} \frac{(-1)^{p+1}}{p^{k}}=\sum_{p=1}^{\infty} \frac{1}{p^{k}}-2 \sum_{p=1}^{\infty} \frac{1}{(2 p)^{k}} .
$$

The right-hand side of this equation may be summed according to [13; p. 169, Eq. (7.1)]

$$
\sum_{p=1}^{\infty} \frac{(-1)^{p+1}}{p^{k}}=\left[1-\frac{1}{2^{k-1}}\right] L i_{k}(1) .
$$

Combining (15) and (17) through (21), a closed form for $E(y)$ is found. For $k$ odd

$$
E(y)=-2 \sum_{\mu=0}^{(k-8) / 2} y^{k-2 \mu}\left[1-\frac{1}{2^{k-2 \mu-1}}\right] L i_{k-2 \mu}(1)-2 y \log 2+\frac{y \Omega(y)}{2}
$$

and for $k$ even

$$
E(y)=-2 \sum_{\mu=0}^{(k-2) / 2} y^{k-2 \mu}\left[1-\frac{1}{2^{k-2 \mu-1}}\right] L i_{k-2 \mu}(1)+\frac{\pi y}{\sin \pi y}-1 .
$$

In (22) the summation on $\mu$ does not exist if $k=1$.

The parametric equations for the desired envelopes may be obtained by using a standard procedure $[17 ;$ p. 85]. Differentiating $(16)$ with respect to $y$, one obtains

$$
B=\frac{E^{\prime}(y)}{C^{\prime}(y)}
$$

Differentiating (14), (22), and (23) and combining them according to (24), we obtain 
(6) and (8). Furthermore, inserting (24) into (16), we have

$$
\xi=\frac{C(y) E^{\prime}(y)}{C^{\prime}(y)}-E(y) \text {. }
$$

Through some elementary manipulations, (25) may be converted into (5) and (7). This completes the proof.

Before leaving this section, it should be noted that in certain cases the upper bounds on a non-monotonic $A(t)$ given by the dotted curves of Fig. 3 can also be strengthened. This is possible if it is known that $A(t)$ satisfies the following conditions.

(a) $A(t)$ is non-decreasing in the interval, $0<t<\tau_{\gamma}$, where $A\left(\tau_{\gamma}\right)<r$.

(b) Beyond $\tau_{\gamma}, A(t)$ remains within the bounds,

$$
A\left(\tau_{\gamma}\right) \leq A(t) \leq 2 r-A\left(\tau_{\gamma}\right) .
$$

In this case the same reasoning used before leads to the following conclusion. In the interval, $0<t<\tau_{\gamma}, A(t)$ is bounded according to that dotted curve of Fig. 2 which has the appropriate $k$. Unfortunately, for a given $Z(s)$ a value of $\tau_{\gamma}$ that satisfies conditions (a) and (b) is not usually known so that this last result is not readily applicable.

Examples. To get an estimate of how close the bounds of the theorem are to being best possible, two examples, were computed for the unit step responses of the subclass 1 functions. The first subclass 1 function is the normalized driving-point impedance of the critically damped, shunt-peaked filter, given by the following expression

$$
Z(s)=\frac{s+4}{(s+2)^{2}} \text {. }
$$

The corresponding unit step response is

$$
A(t)=1-(1+t) e^{-2 t} .
$$

It is plotted as curve (b) in Fig. 4. Curve (a) of Fig. 4 is the upper bound on such unit step responses; i.e., it is the solid curve for $k=1$ in Fig. 3. Any possible improvement in this bound cannot be greater than the amount indicated by curve (b).

It can be noted that the horizontal distance between curves (a) and (b) is greatest in the neighborhood of the final value line. Another example of a non-decreasing unit step response, which is closer to the upper bound in this neighborhood and corresponds to a subclass 1 function, is provided by the following

$$
Z(s)=\frac{(s+2.56)^{2}+(3.56)^{2}}{(s+2.56)\left[(s+2.56)^{2}+1\right]} .
$$

The corresponding unit step response is non-decreasing and is given by

$$
A(t)=1-e^{-2.56 t}[4.98+1.56(\sin t-2.56 \cos t)] .
$$

It is plotted as curve $(c)$ in Fig. 4 and provides a sharper estimate on any possible improvement in curve (a) for $A(t) / r>0.9$.

The moments of non-decreasing unit step responses of subclass $k$ functions. The moments of non-decreasing unit step responses are given by

$$
m_{i}=\frac{1}{r} \int_{0}^{\infty} t^{i} d A(t)
$$




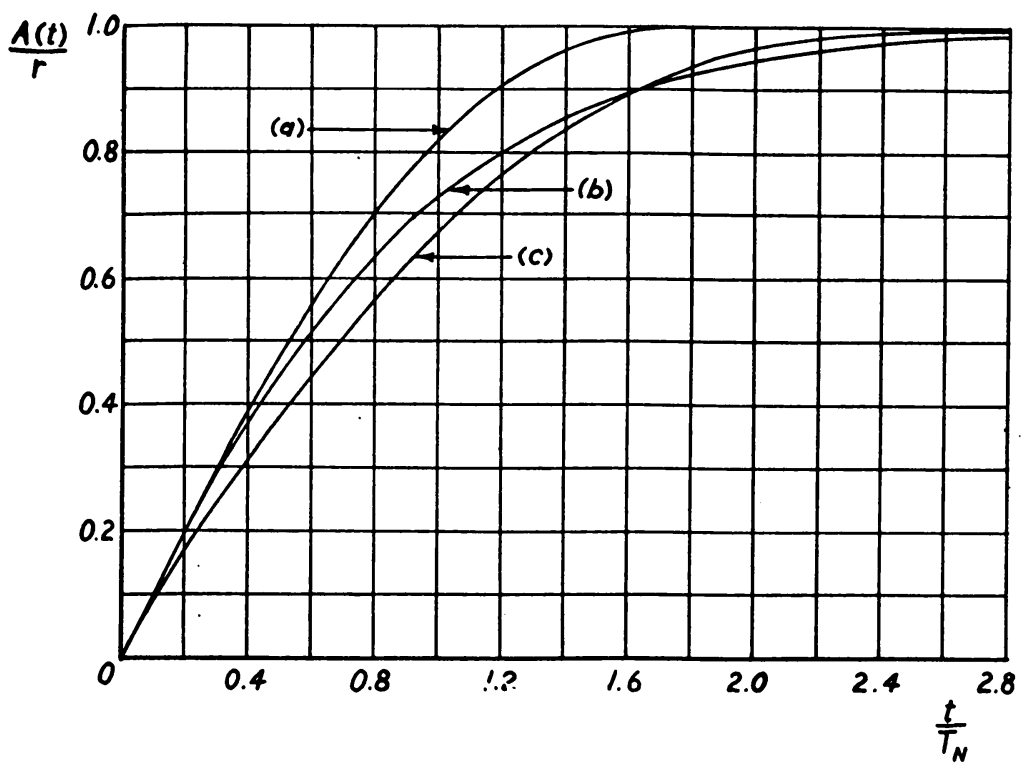

Fra. 4. (a) The upper bound on the monotonic unit step responses of the subclass 1 functions. (b) The unit step response of the shunt-peaked filter given by Eq. (27). (c) The unit step response given by Eq. (29).

where $r=Z(0)=A(\infty)$ and $i=1,2,3, \cdots$. Since $t^{i}$ and $A(t)$ are non-decreasing in $0 \leq t<\infty$, lower bounds on $m_{i}$ may be obtained by replacing $A(t) / r$ by its non-decreasing upper bound $B(y)$. Note that $B(1)=A(\infty) / r=1$. Furthermore, using (4) we have

$$
m_{i}>T_{N}^{i} \int_{0}^{1}[k ! \xi(y)]^{i / k} \frac{d B}{d y} d y
$$

$Z(s)$ is rational so that $A(t) / r$ cannot achieve its upper bound. Hence, equality cannot be achieved in this expression. Here, $\xi(y)$ is given by either (5) or (7) and $d B / d y$ is the derivative of either (6) or (8). Thus, integral expressions for the lower bounds on these movements have been formulated.

\section{ApPENDix}

Parametric Equations for Envelopes Developed ix [1] for the Case Where $A(t)$ Is Not Necessarily Mojotonic

1. The lower bounds on the rise time. As in [1], let us define the rise time $T_{\gamma}$ as the least time where $A\left(T_{\gamma}\right)=r>0$ and beyond which $A(t)$ is bounded by $(1 \pm \gamma) r$, $\boldsymbol{\gamma}$ being a positive number. Expression (36) of [1] gives a family of straight lines which define an envelope that yields a lower bound on $T_{\gamma}$. This expression is repeated here. For $0<y<1$, we have

where

$$
\frac{1}{k !}\left[\frac{T_{\gamma}}{T_{N}}\right]^{k}>-Q(y) \gamma+V(y),
$$

$$
Q(y)=2 y^{k+2} \sum_{p=1}^{\infty} \frac{1}{p^{k}\left(p^{2}-y^{2}\right)},
$$




$$
V(y)=\frac{\pi y}{\sin \pi y}-2 y^{k+2} \sum_{p=1}^{\infty} \frac{(-1)^{p+1}}{p^{k}\left(p^{2}-y^{2}\right)} .
$$

Let $\xi_{\gamma}$ be the lower bound for the left-hand side of (30). Thus,

$$
\frac{T_{\gamma}}{T_{N}}>\left(k ! \xi_{\gamma}\right)^{1 / k}
$$

and (30) becomes

$$
\xi_{\gamma}=-Q(y) \gamma+V(y) .
$$

Proceeding as in $[17 ;$ p. 85$]$, the parametric equations for the envelope of the family of straight lines of (34) are found to be

$$
\begin{gathered}
\gamma=\frac{V^{\prime}(y)}{Q^{\prime}(y)}, \\
\xi_{\gamma}=-\frac{V^{\prime}(y) Q(y)}{Q^{\prime}(y)}+V(y) .
\end{gathered}
$$

The expressions for $Q(y)$ and its derivative may be summed into the following closed forms by using a procedure similar to that employed in summing $E(y)$. For $k$ odd,

$$
\begin{gathered}
Q(y)=-2 \sum_{\mu=0}^{(k-3) / 2} y^{k-2 \mu} L i_{k-2 \mu}(1)-y[\Psi(y)+\Psi(-y)+2 g], \\
Q^{\prime}(y)=-2 \sum_{\mu=0}^{(k-3) / 2}(k-2 \mu) y^{k-2 \mu-1} L i_{k-2 \mu}(1)-\Psi(y) \\
-\Psi(-y)-2 g-y \Psi^{\prime}(y)+y \Psi^{\prime}(-y),
\end{gathered}
$$

where $g$ is Euler's constant $(0.5772 \cdots)$ and where the summation on $\mu$ does not appear if $k=1$. For $k$ even,

$$
\begin{gathered}
Q(y)=-2 \sum_{\mu=0}^{(k-2) / 2} y^{k-2 \mu} L i_{k-2 \mu}(1)+1-\pi y \operatorname{ctn} \pi y \\
Q^{\prime}(y)=-2 \sum_{\mu=0}^{(k-2) / 2}(k-2 \mu) y^{k-2 \mu-1} L i_{k-2 \mu}(1)+\frac{\pi}{\sin \pi y}\left[\frac{\pi y}{\sin \pi y}-\cos \pi y\right] .
\end{gathered}
$$

Furthermore, by comparing (32) with (14) and (15) we see that

$$
V(y)=C(y)-E(y) \text {. }
$$

Hence, for $k$ odd

$$
\begin{array}{r}
V(y)=2 \sum_{\mu=0}^{(k-3) / 2} y^{k-2 \mu}\left[1-\frac{1}{2^{k-2 \mu-1}}\right] L i_{k-2 \mu}(1)+2 y \log 2-\frac{y \Omega(y)}{2}+\frac{\pi y}{\sin \pi y}, \\
V^{\prime}(y)=2 \sum_{\mu=0}^{(k-3) / 2}(k-2 \mu) y^{k-2 \mu-1}\left[1-\frac{1}{2^{k-2 \mu-1}}\right] L i_{k-2 \mu}(1) \\
+2 \log 2-\frac{\Omega(y)}{2}-\frac{y \Omega^{\prime}(y)}{2}+\frac{\pi}{\sin \pi y}(1-\pi y \operatorname{ctn} \pi y),
\end{array}
$$

where $\Omega(y)$ and $\Omega^{\prime}(y)$ are given by (9) and (10), respectively, and where the summation 
on $\mu$ does not exist if $k=1$. For $k$ even

$$
\begin{gathered}
V(y)=1+2 \sum_{\mu=0}^{(k-2) / 2} y^{k-2 \mu}\left[1-\frac{1}{2^{k-2 \mu-1}}\right] L i_{k-2 \mu}(1), \\
V^{\prime}(y)=2 \sum_{\mu=0}^{(k-2) / 2}(k-2 \mu) y^{k-2 \mu-1}\left[1-\frac{1}{2^{k-2 \mu-1}}\right] L i_{k-2 \mu}(1) .
\end{gathered}
$$

Thus, (33) and (35) are the parametric equations for the lower bounds on the rise time where $\xi_{\gamma}$ is given by (36). The quantities in (35) and (36) are given by (37) through (45), where $0<y<1$. For $k$ ranging from one to five, these bounds are plotted in Fig. 3 of [1].

2. The lower bounds on the settling time. The parametric equations for the lower bounds on the settling time may be developed in a similar fashion. From expression (39) of [1], we have for $0<y<1$

$$
\frac{1}{k !}\left[\frac{\tau_{\delta}}{T_{N}}\right]^{k}>-J(y) \delta+V(y)
$$

where $V(y)$ is given by (32) and

$$
J(y)=\frac{\pi y}{\sin \pi y}+2 y^{k+2} \sum_{p=1}^{\infty} \frac{1}{p^{k}\left(p^{2}-y^{2}\right)} .
$$

Letting $\xi_{\delta}$ be the lower bound for the left-hand side of (46), the parametric equations for the lower bounds on the settling time are found to be

$$
\begin{gathered}
\frac{\tau_{\delta}}{T_{N}}>\left(k ! \xi_{\delta}\right)^{1 / k}, \\
\delta=\frac{V^{\prime}(y)}{J^{\prime}(y)},
\end{gathered}
$$

where

$$
\xi_{d}=-\frac{V^{\prime}(y) J(y)}{J^{\prime}(y)}+V(y) .
$$

The closed form expressions for $V(y)$ and $V^{\prime}(y)$ are given by (42) through (45). Furthermore,

$$
\begin{gathered}
J(y)=\frac{\pi y}{\sin \pi y}+Q(y), \\
J^{\prime}(y)=\frac{\pi}{\sin \pi y}(1-\pi y \operatorname{ctn} \pi y)+Q^{\prime}(y),
\end{gathered}
$$

so that the closed form expression for $J(y)$ and $J^{\prime}(y)$ are obtained by combining ( 51 ) and (52) with (37) through (40). For $k$ ranging from one to five, these bounds are plotted in Fig. 4 of [1].

Acknowledgment. The author is indebted to Professors A. Erdélyi and L. Lewin for pointing out how the infinite series in (15) and (31) may be summed. 


\section{REFERENCES}

1. A. H. Zemanian, On transfer functions and transients, Quart. Appl. Math. 16, 273-294 (Oct. 1958)

2. A. H. Zemanian, Some properties of rational transfer functions and their Laplace transformations, Quart. Appl. Math. 17, 245-253 (Oct. 1959)

3. A. H. Zemanian, Further properties of certain classes of transfer functions, Quart. Appl. Math. (to be published)

4. A. H. Zemanian, Generalizations of the concept of the positive real function, IRE Trans. on Circuit Theory CT-6, 374-383 (Dec. 1959)

5. L. Weinberg, Network design by use of modern synthesis techniques and tables, Proc. Natl. Electronics Conf. 12, 794-817 (1956)

6. K. W. Henderson and K. H. Kautz, Transient responses of conventional filters, IRE Trans. on Circuit Theory, CT-5, 333-347 (Dec. 1958).

7. A. Papoulis, Optimum filters with monotonic response, Proc. IRE 46, 606-609 (March 1958)

8. R. C. Palmer and L. Mautner, A new figure of merit for the transient response of video amplifiers, Proc. IRE 37, 1073-1077 (Sept. 1949)

9. I. F. Macdiarmid, Transient response, Wireless Engineer 28, 330-334 (Nov. 1951)

10. O. P. D. Cutteridge, Monotonic transient response, Proc. I. E. E. 101, Part IV, 46-54 (1954)

11. J. D. Brule, Time-response characteristics of a system as determined by its transfer function, IRE Trans. on Circuit Theory CT-6, 163-170 (June 1959)

12. A. H. Zemanian, The properties of pole and zero locations for nondecreasing step responses, Trans. Am. Inst. Elec. Engrs. (to be published)

13. L. Lewin, Dilogarithms and associated functions, Macdonald and Co., London, 1958

14. H. T. Davis, Tables of higher mathematical functions, vols. I and II, The Principia Press, Bloomington, Ind., 1933 and 1935

15. A. H. Zemanian, Restrictions on the shape factors of the step response of positive real system functions, Proc. IRE 44, 1160-1165 (Sept. 1956)

16. A. Erdelyi, W. Magnus, F. Oberhettinger, and F. G. Tricomi, Higher transcendental functions, vol. I, McGraw-Hill Book Co., New York, 1953

17. E. L. Ince, Ordinary differential equations, Dover Publications, New York, 1944 